\title{
BUENOS Y MALOS. SOBRE EL VALOR EPISTÉMICO DE LAS ACTITUDES MORALES Y DE LAS PRUDENCIALES
}

\section{Introducción}

i primer contacto con la filosofía del Derecho académica fue a través de un
iusnaturalista profesor de Derecho Natural de cuyo nombre no quiero
acordarme. El segundo, que además resultó ser decisivo en mi biografía,
fue a través de las clases de Manuel Atienza y del libro de Elías Díaz
Sociología y Filosofía del Derecho. De las clases del primero y del libro del segundo aprendí muchas cosas, pero una de las que más hondo caló en mí -no se si por simple reacción- fue la de las potencialidades del positivismo conceptual frente a otras formas de aproximación al Derecho, principalmente frente al iusnaturalismo. Las tesis centrales de aquel positivismo que entonces estudié se resumían en lo siguiente: a) El Derecho es una institución social, lo que implica que el único Derecho que existe es el positivo, el dado, el socialmente vigente. Y b) la conexión entre el Derecho y la moral es contingente, no necesaria. Las virtudes de dicho enfoque venían dadas por lo siguiente: a) Permitía eludir cualquier tentación metafísica, pues el conocimiento del Derecho, como el de cualquier otra institución social, se resolvía dando cuenta de la conducta y de las creencias de los miembros de la sociedad en cuestión. Y b) al distinguir netamente entre el Derecho que es y el que debe ser, mantenía intacta la capacidad crítica del individuo que reconocía algo como Derecho.

Más adelante, una vez acabada la licenciatura, cuando empezaba a formarme como profesor de filosofía del Derecho, profundicé algo más en la cuestión y, aunque incorporé un sinfín de matices y detalles, esas enseñanzas permanecieron en mí prácticamente inalteradas. Durante algunos años formaron parte no de lo que se discute, sino de aquello a partir de lo que se discute. Pero hoy, esa actitud sólo puede mantenerse al precio de ignorar buena parte de la mejor 
filosofía del Derecho. En efecto, en los últimos años el positivismo «tipo Hart» ha sufrido un auténtico aluvión de críticas: unas como consecuencia de lo que podríamos llamar el desarrollo de algunos elementos presentes en la propia teoría de Hart (principalmente a partir del desarrollo del punto de vista interno y la reelaboración de la teoría desde el modelo del aceptante); otras fruto de un ataque frontal a algunos de sus postulados. Aquí, aunque de forma muy libre, me van a interesar las primeras.

En este trabajo me propongo apuntar algunas ideas muy simples a propósito de la cuestión de la conexión entre Derecho y moral. Para ello, en primer lugar, trataré de esbozar dos teorías del Derecho simétricas pero opuestas con el fin de extraer de ahí algunas conclusiones. En segundo lugar, introduciré un breve excurso sobre el papel que las fuentes del Derecho desempeñan en el razonamiento jurídico justificatorio, lo cual tratará de constituir una breve exposición sobre la racionalidad jurídica. Advierto ya de antemano que me propongo circular por los vericuetos de la teoría del Derecho de forma muy libre y, tal vez, poco rigurosa. A ver qué sale.

\section{Dos teorías del Derecho simétricas, opuestas y parciales}

Cuando se abordan in toto las relaciones entre Derecho y sociedad es común afirmar, al menos desde la tradición conflictualista, que el Derecho desempeña tanto una función ideológica como una función represiva ${ }^{1}$. En correspondencia con lo anterior, cuando se da el salto y se trata de explicar la conducta del sujeto que se adapta a los requerimientos del Derecho se acude o bien a la aceptación ideológica (o consenso) o bien a la presión coactiva, de donde se extraen conclusiones a propósito de la noción de vinculatoriedad, fuerza vinculante u obligatoriedad del Derecho. Si traducimos lo anterior en términos de razones para la acción, nos encontramos con que explicamos la conducta del sujeto por apelación o bien a razones morales o bien a razones prudenciales. En otras palabras, en nuestras explicaciones las que podríamos llamar razones jurídicas quedan reducidas a unas $\mathrm{u}$ otras razones. Ello, sin embargo, no puede sorprender demasiado porque si aceptamos que en los dominios que aquí nos interesan la clasificación de las razones para actuar en morales y prudenciales es exhaustiva, entonces hubiera resultado muy raro que

\footnotetext{
${ }^{1}$ Es obvio que las expresiones «ideología» y «represión» tienen la carga crítica característica del conflictualismo. Sin embargo, en el contexto del presente trabajo me interesa prescindir de dicha carga $\mathrm{y}$, en consecuencia, uso respectivamente esas expresiones en el sentido de consenso y coacción.
} 
las razones jurídicas no hubieran encajado en alguna de ellas. Hasta aquí todo resulta pacífico y no parece haber demasiados problemas. Las dificultades surgen cuando se aborda la «superpregunta» de la teoría del Derecho: qué es esencial al Derecho.

Imaginemos a dos hipotéticos teóricos del Derecho. Para responder a la «superpregunta» ambos recurren al método más fiable que conocen: la observación. Así, observan la realidad social y tratan de obtener datos que les permitan resolver la cuestión. De la observación de la conducta y de las creencias de la gente cada uno de ellos obtiene unos resultados. A partir de ellos tratará de reconstruir los conceptos jurídicos básicos y finalmente abordará la cuestión de las conexiones conceptuales o conexiones necesarias.

\section{Una observación de la realidad y una teoría del Derecho}

El primero de nuestros hipotéticos teóricos resume así sus observaciones: «Es obvio que el Derecho respalda el cumplimiento de sus normas mediante el recurso a la fuerza o la coacción; pero ello es accesorio, no definitorio del mismo. Hay infinidad de situaciones sociales que no identificamos como Derecho en las que requerimientos de conducta aparecen respaldados por amenazas. Lo fundamental es percatarse de que en toda sociedad que cuenta con un orden jurídico hay gente que cumple voluntariamente las normas jurídicas (es decir, no como resultado de las amenazas). Si se observan con detenimiento las creencias de esa gente y se analiza el lenguaje en que las expresan es fácil percatarse de que esas personas muestran una actitud normativa consistente en el aprecio de las normas jurídicas en sí mismas consideradas. Eso quiere decir que para ellos esas normas jurídicas están dotadas de un valor moral. Naturalmente no todos los miembros del grupo social muestran (o tienen que mostrar) esa actitud, pero sí algunos. Especialmente aquellos que detentan mayor poder normativo, esto es, los «funcionarios». Allí donde esa actitud no se detecte no estaremos ante un orden jurídico: será un régimen de pura coacción. En consecuencia, la clave de lo jurídico se encuentra en dicha actitud y la tarea principal del teórico del Derecho consiste en distinguir al Derecho de las situaciones de coacción desnuda».

Nuestro teórico, que, por cierto, está dotado de una gran capacidad analítica, aborda a continuación la tarea de reconstruir los conceptos básicos del Derecho de forma coherente con lo que ha sido el núcleo central de sus observaciones. Para ello decide crear un modelo teórico de participante, al que va a llamar «hombre bueno», cuyas características serán las siguientes: 1 . es un sujeto que cumple 
voluntariamente las normas; 2. lo hace porque tiene hacia ellas una actitud normativa consistente en apreciarlas en sí mismas. Los conceptos jurídicos en manos del hombre bueno adquieren, en consecuencia, una genuina dimensión normativa o práctica. Por ejemplo, a través de él los juicios de validez dejan de ser juicios de deber relativos a un orden, para pasar a ser juicios sobre lo que debe ser; la obligación jurídica queda desvinculada de la coacción o las amenazas; y las autoridades no son sólo sujetos a los que otros reconocen poder normativo sino sujetos que realmente lo tienen, a los que se debe obedecer. En definitiva, resume nuestro teórico, para el hombre bueno las llamadas razones jurídicas se resuelven en razones morales. Ello, por cierto -añadirá- tiene su reflejo en el análisis del razonamiento jurídico justificatorio. En efecto, para que el razonamiento jurídico opere como un razonamiento práctico hay que presuponer una premisa implícita de aceptación de las razones jurídicas, esto es, algo parecido a una premisa que diga que lo establecido por el Derecho debe ser. $\mathrm{O}$ dicho en otras palabras, una premisa que transforme las llamadas razones jurídicas en razones morales. Ello es así porque lo que justifica al sujeto que se conforma a los requerimientos del Derecho no es el hecho de que alguien haya dictado una norma, sino mas bien la creencia en la legitimidad de la misma.

Ahora bien, como ya se ha dicho, el modelo del hombre bueno es el resultado de la observación de la conducta y las creencias de los miembros del grupo. Nuestro observador ha privilegiado a un determinado tipo de conductas y creencias frente a otros tipos que también han sido objeto de observación. Al adoptar este modelo no nos está diciendo que todos sean hombres buenos, sino que lo definitorio del Derecho es la presencia de ese tipo de actitudes, esto es, personas que creen en la legitimidad del orden jurídico y que obran en consecuencia con esa creencia. Si no detectásemos sujetos así no estaríamos ante un orden jurídico. Pero, naturalmente -nos dirá- pueden darse otros tipos de actitudes y de sujetos. En particular, nuestro teórico del Derecho hace la siguiente clasificación no exhaustiva de modelos accesorios, no definitorios: a) El hombre malo: es un calculador preocupado por alcanzar sus propios objetivos dentro del orden jurídico; el Derecho le suministra razones prudenciales y, en consecuencia, actúa motivado por él. b) El delincuente: cree en la legitimidad del Derecho pero carece de la voluntad necesaria para cumplir con sus requerimientos; o bien, carece de creencias morales y se limita a perseguir sus objetivos al margen del Derecho. c) El desobediente: cree en la legitimidad del Derecho, pero incumple una determinada norma porque la considera ilegítima; y d) El rebelde: niega toda legitimidad al orden jurídico.

A partir de todo ello, nuestro observador aborda la cuestión de 
las conexiones necesarias, En primer lugar constata una trivialidad: como cuestión de hecho suele darse una correspondencia entre Derecho y moral positiva. Pero no se queda ahí. Así, dirá que desde una perspectiva conceptual se da una conexión necesaria entre Derecho y moral: si lo característico del Derecho es la presencia de actitudes normativas como las representadas por el hombre bueno, entonces no se puede dar cuenta del Derecho sin dar cuenta de las genuinas creencias morales de esos sujetos. La tesis de la conexión conceptual entre Derecho y moral es un corolario que se presenta como necesario al haber enfocado el Derecho a través de la lente del hombre bueno. Nuestro teórico del Derecho se percata de que la tesis de la conexión conceptual puede formularse en diversos grados, pero decide aplazar para otra ocasión el esclarecimiento de dicha cuestión. Las conexiones que se podrían establecer mirando el Derecho a través de los modelos no definitorios serán obviamente contingentes.

\section{Otra observación de la realidad y otra teoría del Derecho}

Imaginemos que el segundo teórico del Derecho lee el texto en el que el primero ha reflejado las ideas antes expuestas. Al finalizar la lectura comenta a su ayudante: «Nuestro querido colega ha realizado un considerable esfuerzo y creo que ha salido victorioso en su tarea de dar cuenta de los conceptos básicos del Derecho de forma coherente con sus observaciones; pero todo ese esfuerzo ha sido inútil: ha tomado la apariencia por la realidad». Al cabo de unos segundos, añade con voz maledicente: «En realidad, lo único que ha hecho es tomar al tonto por bueno y al listo por malo». A continuación, se encamina hacia el ordenador dispuesto a invertir la teoría de su colega, a asentarla con los pies en el suelo.

Resume de esta manera el resultado de sus observaciones: «Es manifiesto que el Derecho y algunos operadores jurídicos utilizan un lenguaje muy parecido al de la moral, pero ello es completamente accesorio. Dar demasiada importancia a ese lenguaje normativo es confundir la apariencia con la realidad. Es posible que algunos sujetos muestren actitudes morales hacia el Derecho, pero lo relevante es que, a pesar de todo ello, el Derecho, a diferencia de la moral, respalda coactivamente sus requerimientos de conducta. Ello no quiere decir que la única estrategia motivadora del Derecho sean las amenazas; a veces recurre también a la motivación positiva. Lo importante es darse cuenta de que el Derecho suministra razones prudenciales para actuar. La clave de lo jurídico se encuentra, pues, en la noción de fuerza o coacción, y en el razonamiento prudencial vinculado 
a ella. Allí donde los requerimientos de conducta no estén respaldados por la fuerza no podrá hablarse de Derecho. En consecuencia, y debido a la confusión a la que puede llevar el lenguaje moralizante del Derecho, la tarea principal del teórico del Derecho radica en distinguir el Derecho de la moral».

Como este teórico se ha propuesto invertir la teoría del Derecho de su colega, sigue un esquema de razonamiento semejante. Así, privilegia como modelo teórico a través del cual habrá que mirar el Derecho el del «hombre malo», es decir, un calculador racional preocupado por alcanzar sus objetivos dentro del orden jurídico de la forma menos costosa para él. Sus características son las siguientes: 1. adapta su conducta a los requerimientos del Derecho; y 2. lo hace porque éste le motiva prudencialmente. En manos del hombre malo, los conceptos jurídicos adquieren una genuina dimensión prudencial. Por ejemplo, las normas jurídicas pasan a ser instrumentos para la predicción de las consecuencias jurídicas de las conductas; la obligación jurídica queda vinculada a la probabilidad de una sanción en caso de incumplimiento; y las autoridades jurídicas serán sujetos con capacidad para imponer sanciones, etc. Esta dimensión prudencial del Derecho tiene por cierto -dirá nuestro teórico- su reflejo en el análisis del razonamiento jurídico justificatorio. En efecto, para que el razonamiento jurídico opere como un razonamiento prudencial hay que presuponer una premisa implícita de aceptación de las razones jurídicas; esto es, algo parecido a una premisa que diga que lo establecido por el Derecho «tiene que ser». O dicho en otras palabras, una premisa que transforme las razones jurídicas en razones prudenciales. Ello es así porque lo que justifica prudencialmente al sujeto que se conforma a los requerimientos del Derecho no es el hecho de que alguien haya dictado una norma, sino más bien la creencia de que si no la cumple sus intereses se verán afectados negativamente.

Ahora bien, el modelo del hombre malo es el resultado de la observación de la conducta y las creencias de la gente. Nuestro observador lo ha privilegiado frente a otros tipos de actitudes y de sujetos. Al adoptar este modelo no nos está diciendo que todos los miembros de la sociedad sean hombres malos, sino que lo definitorio del Derecho es la presencia de este tipo de sujetos, esto es, gente que cree en la capacidad del Derecho para afectar sus intereses y que obra en consecuencia con esa creencia. Si no hubiera sujetos así, ello querría decir que el orden observado no suministraría razones prudenciales y que, en consecuencia, no sería un orden jurídico. Sería tal vez un orden moral, pero no jurídico. Coherentemente con lo anterior y siguiendo a su colega, nuestro teórico del Derecho hace una clasificación no exhaustiva de modelos accesorios que pueden 
observarse dentro de un orden jurídico. Distingue los siguientes tipos: a) El hombre bueno: es un sujeto que cree en el lenguaje moralizante del Derecho y que conforma su conducta a los requerimientos del Derecho de forma semejante a como la conforma a los de la moral. b) El delincuente: cree que la sanción con la que se le amenaza efectivamente constituye la privación de un bien relevante, pero ello no le motiva porque duda de la capacidad del Derecho en una circunstancia determinada para hacerla efectiva. c) El desobediente: cree en la capacidad general del Derecho para afectar sus intereses, pero rechaza una determinada norma como motivadora de su conducta porque la sanción con que se le amenaza no constituye la privación de un bien en absoluto o, si lo constituye, es un bien que puede calificarse de menor. d) El rebelde: niega toda capacidad del Derecho para afectar negativamente sus intereses; en este último tipo encajaría, por ejemplo, aquél que hiciera un llamamiento a la desobediencia en base a que «no se tiene nada que perder y un mundo por ganar».

Desde ahí, nuestro teórico del Derecho enfrenta la cuestión de las conexiones necesarias. En primer lugar constata una trivialidad: como cuestión de hecho suele darse una correspondencia entre el Derecho y la prudencia positiva, entendida esta última como conjunto de creencias prudenciales efectivamente existentes en una sociedad. Ello es bastante claro -nos dirá- porque un orden que pretendiendo guiar la conducta en base a razones prudenciales estuviera en clara oposición a la prudencia positiva se vería abocado al fracaso. En una sociedad de ateos la amenaza de la excomunión resulta realmente poco motivadora. Pero irá más lejos y nos dirá que desde una perspectiva conceptual se da una conexión necesaria entre Derecho y prudencia: si lo característico del Derecho es el razonamiento prudencial derivado de la amenaza con la privación de bienes, entonces no se puede dar cuenta del Derecho sin dar cuenta de las genuinas creencias prudenciales de esos sujetos. La tesis de la conexión necesaria entre Derecho y prudencia es un corolario que se considera necesario al haber privilegiado al hombre malo; esto es, al haber mirado al Derecho a través de ese peculiar sujeto. Las conexiones que podrían observarse si se mirara al Derecho a través de los modelos por él considerados accesorios, no definitorios, serán, obviamente, contingentes.

\section{Las críticas cruzadas y las simetrías}

No es difícil imaginar las críticas que uno y otro teórico se dirigirían mutuamente. El primero de ellos probablemente diría que la 
teoría del Derecho del segundo es burdamente parcial. Para rechazarla le bastaría con mostrar que con el concepto de Derecho que de ella se obtiene no es posible distinguir a aquellos órdenes sociales que usualmente calificamos como Derecho de cualquier otra situación de coacción realmente existente, dotada de cierta organización y continuidad (por ejemplo, una banda mafiosa). En otras palabras, en su opinión esta teoría del Derecho suministra una imagen distorsionada que priva al Derecho de algo esencial al mismo: la dimensión que lo vincula con la racionalidad práctica.

Por su parte, el teórico del hombre malo acusaría a su colega de igual parcialidad. A él le bastaría para rechazar su concepto de Derecho con mostrar que con él no se podría distinguir entre los órdenes jurídicos existentes y la moral positiva. Y le acusaría de haber dado una imagen angelical del Derecho, tan próxima a la moral positiva, que enmascara toda la dimensión de poder, fuerza y coacción que le parece esencial al mismo.

No creo necesario insistir en las críticas que se dirigirían mutuamente. Me interesa por el contrario mostrar todas las simetrías que hasta ahora he tratado de poner de manifiesto (y alguna más que ha quedado implícita). El siguiente cuadro puede servir de resumen de las mismas:

\begin{tabular}{|c|c|c|}
\hline & Primer teórico & Segundo teórico \\
\hline $\begin{array}{l}\text { Aspectos del D. }{ }^{\circ} \text { como fenómeno } \\
\text { social en que se centra. }\end{array}$ & Fusión ideológica, consenso. & Función represiva, coacción. \\
\hline Razones jurídicas. & Razones morales. & Razones prudenciales. \\
\hline $\begin{array}{l}\text { Participante o modelo } \\
\text { privilegiado. }\end{array}$ & Hombre bueno. & Hombre malo. \\
\hline Modelos marginados. & Junto a otros, el hombre malo. & Junto a otros, el hombre bueno. \\
\hline Pretensión de las autoridades. & Pretensión de legitimidad. & Pretensión de eficacia. \\
\hline $\begin{array}{l}\text { Premisas implícitas en } \\
\text { razonamiento jurídico. }\end{array}$ & $\begin{array}{l}\text { Premisa que convierta e } \\
\text { razonamiento jurídico en uno } \\
\text { moral. }\end{array}$ & $\begin{array}{l}\text { Premisa que convierta e } \\
\text { razonamiento jurídico en und } \\
\text { prudencial. }\end{array}$ \\
\hline Conexión empírica. & Derecho y moral positiva. & Derecho y prudencia positiva. \\
\hline Conexión conceptual. & Derecho y moral. & Derecho y prudencia. \\
\hline Imagen del D. ${ }^{\circ}$ & Próxima a la moralidad positiva. & Próxima a la coacción desnuda. \\
\hline Distorsión que genera. & $\begin{array}{l}\text { Enmascara la dimensión de pode } \\
\text { y coacción del D. }\end{array}$ & $\begin{array}{l}\text { Enmascara la dimensión práctica } \\
\text { del D. }\end{array}$ \\
\hline
\end{tabular}




\section{Las críticas que no pueden dirigirse}

Ninguno de ellos puede reprochar al otro el que se haya limitado a observar conducta social externa. Ambos han tratado de dar cuenta de creencias de la gente. No en vano ambos han operado con el modelo del hombre bueno y con el del hombre malo. Y referirse a ellos presupone, entre otras cosas, distinguir entre conducta simplemente conforme con los requerimientos del Derecho y conducta guiada por el Derecho. Luego ninguno de ellos puede acusar al otro de haber realizado una observación pobre, poco explicativa y superficial. El objetivo de ambos ha sido dar cuenta de las creencias de un participante del sistema.

Tampoco pueden acusarse de haberse comprometido de forma tal que la crítica al Derecho existente se haya vuelto imposible. Para el primero (que, recordemos, llegó a la conclusión de la conexión conceptual necesaria entre Derecho y moral) la crítica del Derecho positivo es siempre posible porque la tesis que él ha sostenido es relativa a las creencias de otros. Considerar la existencia de hombres buenos como el criterio conceptual que nos permitirá distinguir al Derecho de otros fenómenos sociales puramente coactivos no significa elevar al hombre bueno a la categoría de medida objetiva de lo justo. Tampoco se ha comprometido críticamente el teórico que llegó a la conclusión de la conexión necesaria entre Derecho y prudencia. No se olvide que en este dominio la oposición entre el Derecho que es y el que debe ser también funciona. No es difícil observar que una constante de la discusión jurídica es la revisión de los instrumentos de motivación. Pues bien, el hombre malo es portador de ciertas creencias prudenciales pero no es ni mucho menos la medida de la prudencia esclarecida. Que el hombre bueno y el hombre malo estén comprometidos no compromete a los observadores que los han privilegiado. En el fondo, así formuladas, las tesis de las conexiones necesarias son casi triviales. Además, naturalmente, nada impide que tanto el teórico del hombre bueno como el del hombre malo puedan formular críticas al Derecho positivo desde los dominios por ellos no considerados definitorios. El teórico del hombre malo puede formular críticas morales al Derecho positivo y el del hombre bueno críticas prudenciales.

Finalmente, tampoco pueden reprocharse el que no se hayan dado cuenta de que un sujeto puede usar un código de conducta de forma distinta al uso que se deriva de su aceptación colectiva y que conforma su naturaleza. Por ejemplo, es obvio que un sujeto puede usar prudencialmente un código de moralidad positiva sin que ello altere la naturaleza moral de dicho código; o, a la inversa, que un sujeto puede elevar a la categoría de moral un código puramente prudencial (la conducta ritualista, por cierto, tiene bastante que ver 
con ello). Dicha posibilidad no puede negarse porque, por decirlo de algún modo, es consustancial al fenómeno de la positividad. Pues bien, nuestros teóricos han sido perfectamente conscientes de ello y así han privilegiado al sujeto cuya aceptación conforma la naturaleza del código. Junto a él han definido tipos accesorios que no alteran dicha naturaleza.

\section{Una opinión y una hipótesis}

En mi opinión, los dos teóricos aciertan claramente al menos en una cosa: en la crítica que cada uno de ellos dirige a la imagen del Derecho que se sigue de la teoría del otro. En efecto, me parece obvio que tanto la imagen angelical como la de la coación desnuda son parciales y distorsionadoras.

Pero tratemos de comprometernos algo más. Imaginemos que ambos teóricos aciertan parcialmente en la cuestión de las conexiones que han puesto de manifiesto. Es decir, imaginemos que el Derecho mantiene una conexión necesaria con la moral y con la prudencia. Ello supone considerar que los órdenes jurídicos cuentan con dos tipos de participantes: los morales y los prudenciales (el hombre bueno y el hombre malo). No se trata, por consiguiente, de repetir lo que ya han hecho nuestros teóricos: ellos definían un único participante -el hombre bueno o el hombre malo- en correspondencia con la naturaleza normativa o prudencial del Derecho; de forma que los otros sujetos, los que usaban el código de manera distinta a la que se seguía de su naturaleza, no eran participantes y, en consecuencia, tenían un carácter accesorio. La hipótesis, por el contrario, consiste en que los órdenes que llamamos jurídicos cuentan con dos tipos de participantes: los normativos y los prudenciales. La intuición en la que se basa esta hipótesis es la siguiente: un orden de conducta que no contase con participantes del tipo del hombre bueno sería un régimen de pura coacción; y un orden en el que no los hubiera del tipo del hombre malo sería un orden que no suministraría razones prudenciales y, en consecuencia, estaría muy alejado de los órdenes que usualmente llamamos jurídicos.

Pero vayamos por partes y tratemos primero de explorar la plausibilidad de la hipótesis en relación con lo que para cada uno de nuestros teóricos era trivial: me refiero a las conexiones empíricas. La hipótesis presupone que de hecho se da una correspondencia entre Derecho y moral positiva, por un lado, y entre Derecho y prudencia positiva, por otro. Entonces surge de forma prácticamente inadvertida una pregunta que podría formularse en términos cuasicuantitativos: ¿cuál de esas dos conexiones es de hecho más importante? En este punto, y en relación con las conexiones empíricas, tal 
vez pudiera formularse una ley universal: en los órdenes jurídicos la importancia fáctica de la dimensión normativa y de la prudencial depende del grado de cohesión social. En otras palabras, a menor grado de conflicto social, mayor peso de la aceptación ideológica (y del razonamiento normativo vinculado a ella) y menor protagonismo del recurso a la fuerza (y del razonamiento prudencial vinculado a ella). Y a la inversa, a mayor grado de conflicto social, menor peso de la aceptación ideológica (y del razonamiento normativo) y mayor de la coacción (y del razonamiento prudencial).

Pero nuestros autores no sostienen sólo una tesis empírica, sino también una tesis conceptual. La plausibilidad de la tesis de la conexión necesaria entre Derecho y moral, por un lado, y Derecho y prudencia, por otro, puede mostrarse sencillamente a partir de la radicalización de nuestra presunta ley universal. En efecto, si observamos una comunidad fuertemente integrada en la que la aceptación ideológica de las normas de conducta es tan intensa que lleva, por ejemplo, a fenómenos tales como que la desviación se resuelve mediante el autocastigo, difícilmente diríamos que dicha comunidad cuenta con un orden jurídico. Y de igual modo, si la comunidad observada presentase un nivel de conflicto tal que hubieran quedado alienados los elementos de consenso, difícilmente diríamos que en esa comunidad rige un orden jurídico.

Para la aceptación de la hipótesis nos quedarían muchas cosas por explicar. Dos me parecen especialmente importantes y, por razones de espacio, a ellas me voy a limitar: la primera es cómo repercute esa doble naturaleza (normativa y prudencial) del Derecho en el razonamiento jurídico justificatorio; y la segunda, desde qué dimensión deben reconstruirse los conceptos jurídicos básicos. Para tratar de apuntar una vía de respuesta a estas preguntas voy a referirme a las fuentes del Derecho.

\section{Las fuentes del Derecho}

La expresión «fuentes del Derecho», incluso tomada exclusivamente en su sentido formal, está preñada de ambigüedades. Kelsen, quien, por cierto, propuso abandonar dicha expresión debido a su carácter equívoco, destacó dos significados posibles de «fuentes del Derecho». El primero es el de modos o métodos de producción de Derecho, y que aquí, con el ánimo de ser algo más concretos, especificaremos en actos o hechos de los que depende la creación de normas jurídicas. El segundo es el de normas superiores en relación con las normas inferiores cuya producción regulan, y que aquí llamaremos normas sobre la producción jurídica. Lo que ocurre es que, en 
cierto sentido, ambos conceptos de fuentes del Derecho se implican recíprocamente, pues si hay actos o hechos productores de Derecho es porque hay normas jurídicas que los califican como tales y si hay normas sobre la producción jurídica es para regular los hechos o actos productores de Derecho. Por ello, me parece que una buena definición de fuentes del Derecho puede ser la siguiente: fuentes del Derecho son los hechos o actos de los que, en virtud de las reglas sobre la producción jurídica, depende la creación o la existencia de las normas jurídicas.

Ahora bien, en el lenguaje jurídico las expresiones que se utilizan para designar a los hechos o a los actos jurídicos presentan una genuina ambigüedad proceso/producto. Si ello es así, es fácil darse cuenta de que las expresiones que utilizamos para referirnos a los diferentes actos o hechos productores de Derecho presentan esa misma ambigüedad proceso/producto. Así, por ejemplo, la expresión «legislación» sirve tanto para referirse al acto de legislar, esto es, al acto de crear un determinado tipo de norma jurídica (proceso), como al resultado (producto) de realizar dicho acto, esto es, a la ley. Para romper esta ambigüedad, conviene distinguir entre fuentes-proceso y fuentes-producto.

Pues bien, a pesar de las dificultades que comporta el concepto de «fuentes del Derecho», me parece difícil que pueda negarse que éstas cumplen una función de certeza y una función justificatoria. La primera, simplificando mucho, podría decirse que opera del siguiente modo: las fuentes-proceso, que constituyen algo parecido al pedigree de las fuentes-producto, permiten determinar con relativa precisión las normas jurídicas y, en consecuencia, los derechos, deberes, poderes, etc., de los individuos; por ello permiten también predecir las consecuencias jurídicas de las conductas.

La función justificatoria opera de la siguiente manera. Si admitimos que el razonamiento jurídico justificatorio típico consta de una premisa normativa (una norma jurídica), una premisa fáctica (un enunciado empírico) y una conclusión normativa (una norma particular), podríamos decir que las fuentes del Derecho sirven, entre otras cosas, para la justificación externa de la premisa normativa. Es decir, mostrando que ella tiene su origen en una fuente se justifica dicha premisa. Si ello es así, no es difícil aceptar que una de las funciones de las fuentes del Derecho es la de cerrar el razonamiento jurídico justificatorio.

¿Qué quiere decir todo ello? Sencillamente que desde los criterios del orden son irrelevantes las razones por las cuales los sujetos aceptan o usan una determinada premisa normativa. Ello no implica que estemos inaugurando las razones jurídicas como una nueva categoría de razones para actuar distinta de las morales o de las prudenciales. 
El sujeto, como no podría ser de otra manera, seguirá obrando por razones morales o prudenciales. Lo que trato de decir es que el Derecho, de acuerdo con la doble naturaleza de nuestra hipótesis, es un orden, compatible con ambos tipos de razones y que ello se muestra claramente en el hecho de que éste rebaja un grado o un escalón sus exigencias justificatorias en relación con lo que sería un razonamiento práctico o prudencial acabado. El razonamiento jurídico justificatorio no exige la adhesión normativa interna ni el reconocimiento de la eficacia de sus amenazas. Y ello, sostiene la hipótesis, es válido para cualquier sujeto normativo: tanto para los funcionarios que ocupan las más altas magistraturas como para el último de los ciudadanos cuyo poder normativo no va más allá de la capacidad para firmar contratos. El uso de las fuentes del Derecho justifica jurídicamente las decisiones de los magistrados del Tribunal Supremo independientemente de si recurren a ellas porque lo consideran un deber moral, como si lo hacen porque quieren evitar el rechazo social que generaría el que no las tuvieran en cuenta. En eso, la situación de los magistrados es idéntica a la del ciudadano que paga sus impuestos: su conducta está justificada jurídicamente con independencia de que lo haga porque «Hacienda somos todos» o porque quiere evitar la sanción. En todos esos casos se usa el Derecho para justificar conductas y se usa, según la hipótesis, de forma conforme a las razones que él pretende suministrar. Naturalmente, de las fuentes a la regla de reconocimiento hay un paso y no se ve muy bien por qué lo que puede valer para las primeras no pudiera valer para la segunda. En otras palabras, respecto de la regla de reconocimiento habrá también dos tipos de aceptantes: los morales y los prudenciales.

La hipótesis de la doble naturaleza en la existencia social del Derecho se manifiesta, pues, básicamente en que éste rebaja en un grado sus exigencias justificatorias en relación con lo que son los razonamientos prácticos y prudenciales acabados. Otra forma de decir lo mismo es la siguiente: desde las exigencias justificatorias del orden jurídico tanto las razones morales como las prudenciales por las cuales un sujeto pueda usar una norma jurídica pertenecen al contexto de descubrimiento, esto es, son irrelevantes en términos jurídico-justificatorios.

Finalmente, si la hipótesis fuera cierta, tal vez permitiría explicar dos fenómenos persistentes de la reflexión jurídica. Primero, el que convivan sin que pueda hablarse de superación dos reconstrucciones plausibles de los conceptos jurídicos básicos: una normativa y otra prudencial. Segundo, el empeño que los juristas han mostrado por reivindicar un método propio: ese empeño tal vez no sea más que pretender hacer la correspondiente rebaja en un grado en su discurso: emitir juicios según o desde un orden jurídico, sin más. 
$\triangle$

DOXA 15-16 (1994) 